McGill/96-19

\title{
Multiple Reggeon Exchange from Summing QCD Feynman Diagrams
}

\author{
Y.J. Feng ${ }^{\dagger}$ and C. S. Lam* \\ Department of Physics, McGill University, \\ 3600 University St., Montreal, P.Q., Canada H3A 2T8
}

\begin{abstract}
Multiple reggeon exchange supplies subleading logs that may be used to restore unitarity to the Low-Nussinov Pomeron, provided it can be proven that the sum of Feynman diagrams to all orders gives rise to such multiple regge exchanges. This question cannot be easily tackled in the usual way except for very low-order diagrams, on account of delicate cancellations present in the sum which necessitate individual Feynman diagrams to be computed to subleading orders. Moreover, it is not clear that sums of high-order Feynman diagrams with complicated criss-crossing of lines can lead to factorization implied by the multi-regge scenario. Both of these difficulties can be overcome by using the recently developed nonabelian cut diagrams. We are then able to show that the sum of $s$-channel-ladder diagrams to all orders does lead to such multiple reggeon exchanges.
\end{abstract}

\section{Introduction}

The gluon in QCD reggeizes in the leading-log approximation. The coupling constant $(g)$ and the energy $(\sqrt{s})$ of sum of one-reggeized-gluon (1rg) diagrams come in the form $g^{2}\left(g^{2} \ln s\right)^{p}$, where $g^{2} \ll 1, g^{2} \ln s=O(1)$, and $p$ is a non-negative integer. The sum of $2 \mathrm{rg}$ diagrams are of the form $g^{4}\left(g^{2} \ln s\right)^{p}$, and more generally the $m \mathrm{rg}$ amplitude is given by sums of terms of the form $g^{2 m}\left(g^{2} \ln s\right)^{p}$.

For quark-quark elastic scattering at high energy and fixed momentum transfer, the color exchanged in a $1 \mathrm{rg}$ amplitude is an octet, and that for a $2 \mathrm{rg}$ amplitude is either an octet or a singlet. The $2 \mathrm{rg}$ amplitude being a factor $g^{2} \ll 1$ down from the $1 \mathrm{rg}$ amplitude, its octet contribution can be neglected, but its singlet part must be kept, for there is no competing contribution from the $1 \mathrm{rg}$ amplitude. This singlet part is just the Pomeron proposed by Low and Nussinov [1] .

The leading-log Pomeron amplitude obtained this way [2, 3] violates unitarity. It leads to a total cross section with a power growth in $s$, which is forbidden by the Froissart bound. To unitarize the BFKL equation [2] it is therefore necessary to include subleading-log contributions. 


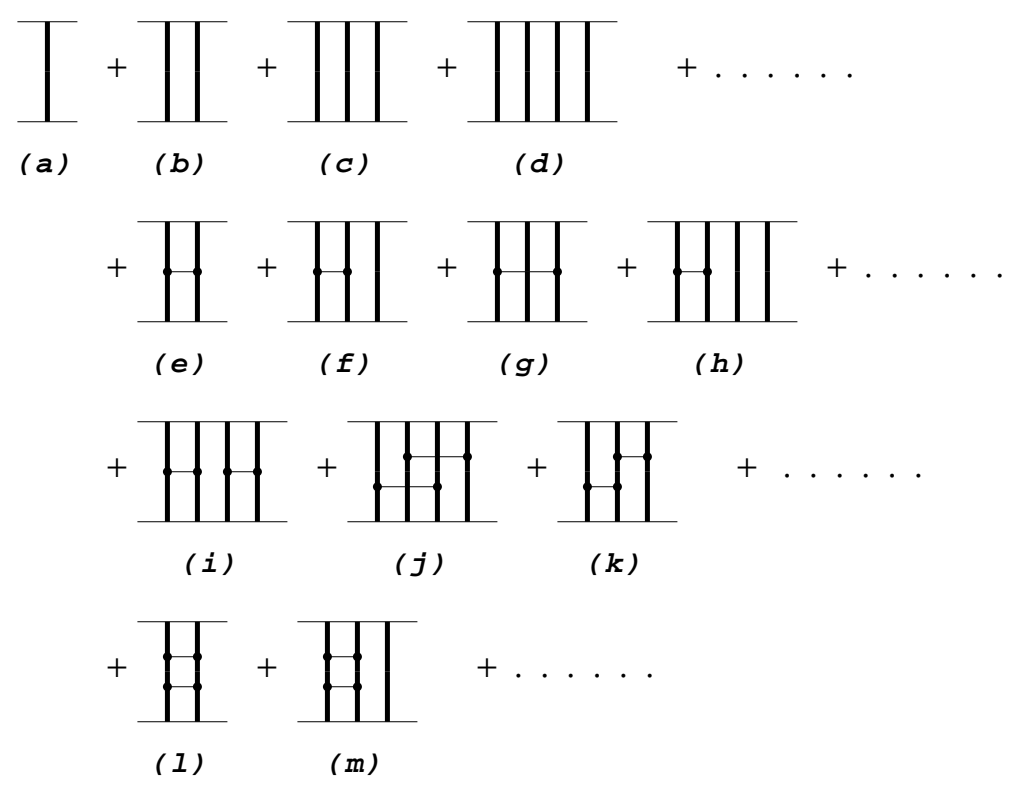

Figure 1: Multi-reggeon exchange diagrams.

Subleading logs are notoriously difficult to extract from Feynman diagrams. One may try to compute some low-order diagrams to get information [4] but it is almost certain that this cannot be carried out to all orders. However, subleading-log contributions to the Pomeron do not necessarily require subleading logs from sums of Feynman diagrams. For example, leading-log calculation of Feynman diagrams contributing to $2 \mathrm{rg}$ exchanges gives subleading-log correction to the octet amplitude. Hence there is a hope in unitarizing the Pomeron amplitude without having to invoke difficult subleading-log calculations. In fact, $s$-channel unitarity in all color channels is formally satisfied if all multiple rg exchanges are added in, as shown in the reggeized diagrams in Fig. 1. To allow for shadows produced by inelastic scatterings, we must include production of gluons from the rg's in the intermediate states, though creation of quark pairs will be ignored in the present discussion. Whether this proposal of unitarization [3], without including subleading terms in individual sums of Feynman diagrams, is correct or not remains an open question which we simply cannot discuss until more is known.

The necessity of including multiple-rg exchange diagrams can be understood in a completely different way, totally within the framework of leading-log approximations. Imagine we are dealing with an $S U\left(N_{c}\right)$ color theory in which quarks carry an arbitrary color. Then there are many independent color amplitudes, more so if $N_{c} \gg 1$ and quarks carrying a large color. To retain the leading-log contribution of every one of these independent color amplitudes, we must retain the $m$ rg contributions for every $m$. So even staying within leading logs, those multiple-rg exchanges are required for a color $S U\left(N_{c}\right)$ theory with arbitrary quark colors. For that reason we shall carry out our calculations below for an arbitrary $N_{c}$ 
and every quark color. Since spin is unimportant in high energy scattering [2, 3], this has the added advantage that whatever we obtain is automatically valid for gluon-gluon scattering as well.

What is missing in this scheme is the proof that the reggeized factorization hypothesis is indeed correct, that the sum of Feynman diagrams in the leading-log approximation does factorize into sums of these multiple-rg amplitudes.

To be sure, the hypothesis has been verified explicitly up to the 6th order, and partially up to the 8th and 10th orders [2, 5, 6], but because of the presence of delicate cancellations, it is difficult to carry similar calculations to higher orders. In fact, these delicate cancellations have not been completely verified even in the 8th and the 10th orders.

The problem is the following. In Feynman gauge calculation which we shall adopt throughout, the leading-log contributions in some color amplitudes get cancelled out when several Feynman diagrams are summed [0, 6, 7]. Consequently, to compute the sum of diagrams to leading-log accuracy, we need to calculate individual diagrams to subleading-log precision. Occasionally this can be accomplished without much pain by using $s \leftrightarrow u$ symmetry, but more often not. To the extent that subleading logs are very difficult to compute, calculations to higher-order diagrams do appear to be quite forbidding.

Even if we manage to overcome this hurdle, the verification of reggeized factorization from sums of Feynman diagrams, with lines crossing one another in very complicated ways, would still seem to be extremely difficult.

Fortunately there is a chance to overcome both of these difficulties by using nonabelian cut diagrams [7] in place of the usual Feynman diagrams. Nonabelian cut diagrams are resummations of Feynman diagrams with these delicate cancellations removed, so that each of them can be computed just in the leading-log approximation. Moreover, factorization is natural to the nonabelian cut diagrams, because they are the graphical manifestation of a 'multiple commutator formula', which in turn was derived from a 'factorization formula' [8], and it is this same factorization formula that will be used to demonstrate reggeized factorization hypothesis for a class of diagrams.

Nonabelian cut diagrams will be reviewed in the next section. Some of their properties, including the assertion of the absence of delicate cancellations in these cut diagrams but their presence in usual Feynman diagrams, will be discussed in Sec. 3. In this paper we shall study in detail, and be able to prove, the reggeized factorization hypothesis for a particular simple class of diagrams, the $s$-channel-ladder diagrams. It is this class of diagrams in QED that can be summed up into an explicit eikonal form, so one would expect it to be the simplest set to study in QCD as well for multiple-rg exchanges and unitarization. However, with color complication, the QCD case is much harder to deal with than the QED case, the details of which are discussed in Sec. 4. For more complicated nonabelian cut diagrams in QCD, we are not yet able to prove the reggeized factorization, but the success for the $s$-channel-ladder diagrams is encouraging. Finally, a short summary and outlook are provided in Sec. 5. 


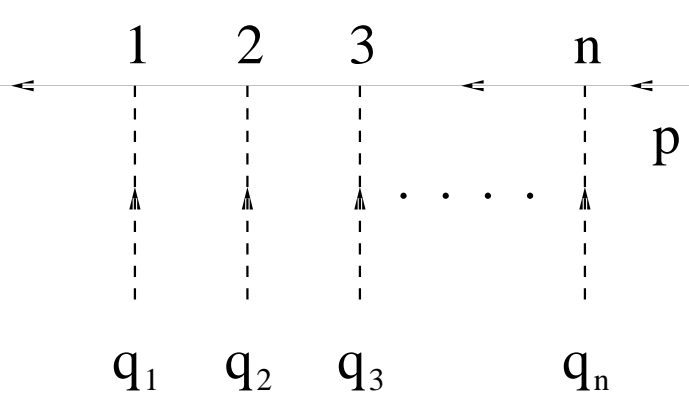

Figure 2: A tree diagram with $n$ bosons emitted or absorbed.

\section{Nonabelian cut diagrams}

Nonabelian cut diagrams [7] represent a resummation of Feynman diagrams. They are not the same as Cutkosky cut diagrams which compute discontinuities of single Feynman diagrams.

A nonabelian cut diagram differs from a Feynman diagram in having certain 'high speed' propagators cut. The cut lines occur among those carrying a large momentum $p$, with comparatively small amount of momenta $q_{i}$ transferred away at each interaction vertex. In that case, the approximation

$$
\left(p+\sum_{j=1}^{i} q_{j}\right)^{2}-m^{2} \simeq 2 p \cdot \sum_{j=1}^{i} q_{j} \equiv \sum_{j=1}^{i} \omega_{j}
$$

is valid, so the denominators of the Feynman propagators for these lines are simplified to $\left(\sum_{j=1}^{i} \omega_{j}+i \epsilon\right)^{-1}$.

Within this approximation, the QCD tree diagram for a propagating quark shown in Fig. 2 is

$$
-2 \pi i \delta\left(\sum_{j=1}^{n} \omega_{j}\right)\left(\prod_{i=1}^{n-1} \frac{1}{\sum_{j=1}^{i} \omega_{j}+i \epsilon}\right) \cdot t_{1} t_{2} \cdots t_{n} \cdot V \equiv a[12 \cdots n] \cdot t[12 \cdots n] \cdot V
$$

where $t_{i}$ are the color matrices of the quark, and $t[12 \cdots n] \equiv t_{1} t_{2} \cdots t_{n}$. The numerator with the normalization convention $\bar{u} u=1$ can be approximated by

$$
V=\frac{1}{2 M} \prod_{i=1}^{n}\left(2 p^{\mu_{i}}\right)
$$

where $\mu_{i}$ are the Lorentz indices of the gluons and $M$ is the quark mass.

The tree diagram in Fig. 2 will be denoted by $[12 \cdots n]$, according to the order of the gluons. If the gluons are labelled differently, say as $\left[\sigma_{1} \sigma_{2} \cdots \sigma_{n}\right]$, then the corresponding spacetime amplitude and color factor will be similarly designated as $a\left[\sigma_{1} \sigma_{2} \cdots \sigma_{n}\right]$ and $t\left[\sigma_{1} \sigma_{2} \cdots \sigma_{n}\right]$. 
Before discussing the nonabelian cut diagrams it is necessary to introduce some notations. If $\left[T_{i}\right]$ are tree diagrams, then $\left[T_{1} T_{2} \cdots T_{A}\right]$ represents the tree diagram obtained by merging these $A$ trees. For example, if $\left[T_{1}\right]=[123]$ and $\left[T_{2}\right]=[45]$, then $\left[T_{1} T_{2}\right]=[12345]$. The notation $\left\{T_{1} ; T_{2} ; \cdots ; T_{A}\right\}$, on the other hand, is used to denote the set of all tree diagrams obtained by interleaving the trees $T_{1}, T_{2}, \cdots, T_{A}$ in all possible ways. This set contains $\left(\sum_{a} n_{a}\right) ! / \prod_{a} n_{a}$ ! trees if $n_{a}$ is the number of gluon lines in the tree $T_{a}$. In the example above, $\left\{T_{1} ; T_{2}\right\}$ contains the following $5 ! / 3 ! 2 !=10$ trees: [12345], [12435], [12453], [14235], [14253], [14523], [41235], [41253], [41523], and [45123].

Correspondingly, $a\left\{T_{1} ; T_{2} ; \cdots ; T_{A}\right\}$ will represent the sum of the amplitudes $a[T]$ for every tree $T$ in this set.

The nonabelian cut diagram [7] is derived from the the multiple commutator formula [8], which states that

$$
\sum_{\sigma \in S_{n}} a[\sigma] t[\sigma]=\sum_{\sigma \in S_{n}} a[\sigma]_{c} t[\sigma]_{c}^{\prime}
$$

This is a resummation formula for the nonabelian tree amplitude (2.2), summed over all $n$ ! permutations $[\sigma] \equiv\left[\sigma_{1} \sigma_{2} \cdots \sigma_{n}\right]$ of $[12 \cdots n]$. The spacetime part of the cut amplitude $a[\sigma]_{c}$ is obtained from the cut diagram $[\sigma]_{c}$, and the color factor $t[\sigma]_{c}^{\prime}$ is obtained from the complementary cut diagram $[\sigma]_{c}^{\prime}$. All of these will be explained below.

The multiple commutator formula in turn was derived from the factorization formula [8], which states that

$$
a\left\{T_{1} ; T_{2} ; \cdots ; T_{A}\right\}=\prod_{a=1}^{A} a\left[T_{a}\right] .
$$

This is a sum rule expressing factorization of sums of certain tree amplitudes. It is this same formula that proves to be invaluable in showing the reggeized factorization later.

A special case of the factorization formula is well known. If $n_{a}=1$ for every $a$ so that the tree $\left[T_{a}\right]=[a]$ is simply a vertex, then $\{1 ; 2 ; \cdots ; A\}$ is the set of $A$ ! permutation of the tree $[12 \cdots A]$, and the factorization formula is just the well-known eikonal formula [9].

We shall now proceed to define the cut diagrams and the cut amplitudes. To each Feynman tree diagram $[\sigma]=\left[\sigma_{1} \sigma_{2} \cdots \sigma_{n}\right]$ of the type shown in Fig. 2, we associate with it a cut diagram $[\sigma]_{c}$ by putting cuts on specific fermion propagators as follows. Proceed from left to right, put a cut after a gluon if and only if a smaller number does not occur to its right. Continuing thus until reaching the end of the tree, and we get the cut diagram. An external line would be considered equivalent to a cut so there is never an explicit cut put in at the end of the tree.

The written notation for a cut will be a vertical bar behind a gluon. Using that notation, here are some illustrations of where cuts are put into Feynman trees: $[1234]_{c}=[1|2| 3 \mid 4]$, $[3241]_{c}=[3241]$, and $[2134]_{c}=[21|3| 4]$.

The complementary cut diagram $[\sigma]_{c}^{\prime}$ is one where lines cut in $[\sigma]_{c}$ are not cut in $[\sigma]_{c}^{\prime}$, and vice versa. Thus $[1234]_{c}^{\prime}=[1234],[3241]_{c}^{\prime}=[3|2| 4 \mid 1]$, and $[2134]_{c}^{\prime}=[2 \mid 134]$. 
The spacetime part of the cut amplitude, $a[\sigma]_{c}$, is simply the Feynman amplitude $a[\sigma]$ with the cut propagator taken to be $-2 \pi i \delta\left(\sum_{j} \omega_{j}\right)$ instead of the usual $\left(\sum_{j} \omega_{j}+i \epsilon\right)^{-1}$. In this way it is the same cut propagator as in the Cutkosky cut diagram, but here cuts are placed only on high speed fermion lines, and as (2.4) indicates, the nonabelian cut diagrams represent a resummation and not a discontinuity.

The color factor $t[\sigma]_{c}^{\prime}$ is determined from the complementary cut diagram $[\sigma]_{c}^{\prime}$. It is obtained from $t[\sigma]$ by replacing the product of color matrices separated by cuts with their commutators. For example, $t[1234]_{c}^{\prime}=t[1234]=t_{1} t_{2} t_{3} t_{4}, t[3214]_{c}^{\prime}=t[3|2| 4 \mid 1]=\left[t_{3},\left[t_{2},\left[t_{4}, t_{1}\right]\right]\right]$, and $t[2134]_{c}^{\prime}=t[2 \mid 134]=\left[t_{2}, t_{1}\right] t_{3} t_{4}$.

A Feynman diagram for quark-quark scattering can be obtained by connecting two trees like Fig. 2 together via the gluon lines, perhaps with the help of triple gluon and four gluon vertices and other propagators in between. Since (2.4) is valid for offshell gluons, it can be applied to one of the two quark trees carrying large momentum even though it is tied up in a loop diagram. Unless otherwise stated, relation (2.4) will always be applied to the upper quark tree, so cuts are normally made only on this line. The rest of the propagators remain uncut and the diagram is otherwise the same as an ordinary Feynman diagram.

\section{Color factors}

We define regge color factors to be the color factors appearing in the reggeized diagrams of Fig. 1. If the reggeized factorization hypothesis is correct, only regge color factors can be present when all Feynman diagrams are summed.

We define primitive color factors as regge color factors that cannot be pulled apart into smaller units by sliding along the pair of quark lines. In other words, they are those that remain connected after the upper and lower quark lines are removed. Thus 1(a), 1(e), 1(k), 1(l) are primitive, but none of the others in Fig. 1 are.

It is shown in App. A that in the leading-log approximation, every regge color factor that has the same number of primitive color units can be considered to be the same, irrespective of how the units are placed. Thus $1(\mathrm{f})$ is the same as $1(\mathrm{~g})$, and $1(\mathrm{i})$ is the same as $1(\mathrm{j})$. The number of times $f_{\alpha}$ a primitive color factor $\phi_{\alpha}$ occurs completely specifies a regge color factor, which will be denoted by $\Phi=\prod_{\alpha} \phi_{\alpha}^{f_{\alpha}}$.

The primitive color factor in 1(a) will be designated as I. The primitive color factor for 1(e) will be designated as $\mathrm{H}$. The regge color factors of $1(\mathrm{~b}), 1(\mathrm{c}), 1(\mathrm{~d}), 1(\mathrm{f}), 1(\mathrm{~g})$, 1(h), 1(i), 1(j) are then $I^{2}, I^{3}, I^{4}, H I, H I, H I^{2}, H^{2}, H^{2}$. In the notation of Refs. [5,7], $I=\mathbf{G}_{\mathbf{1}}, H=\mathbf{G}_{\mathbf{3}}, I^{2}=\mathbf{G}_{\mathbf{2}}$, and $I^{3}=\mathbf{G}_{\mathbf{4}}$.

The color factor of an ordinary Feynman diagram can be decomposed into sums of regge color factors [3, 5] by using the commutation relation

$$
\left[t_{a}, t_{b}\right]=i f_{a b c} t_{c}
$$

as well as the sum rules

$$
f_{a b c} f_{a b d}=2 c \delta_{c d}, \quad i^{3} f_{a d g} f_{b e d} f_{c g e}=c i f_{a b c},
$$




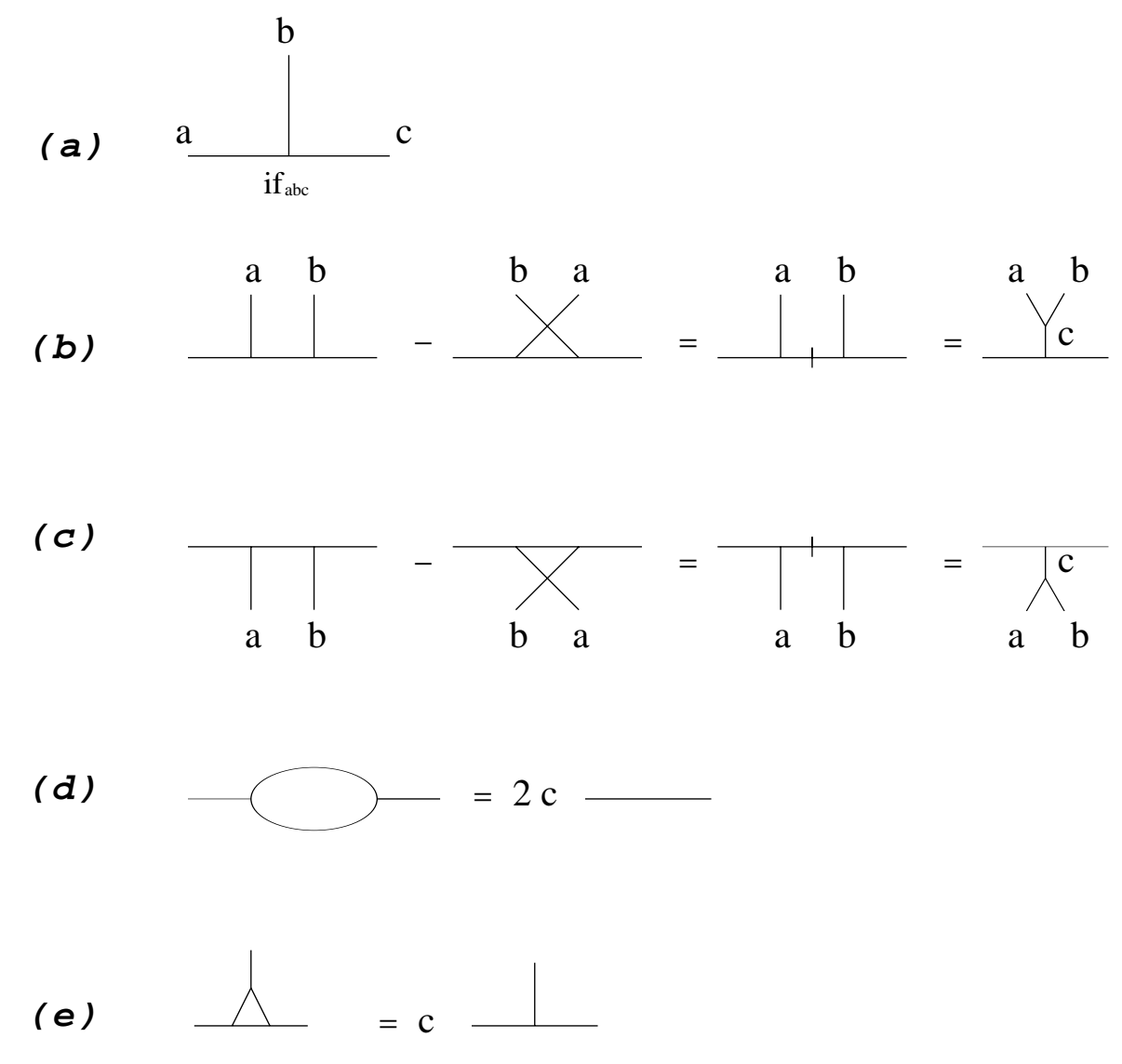

Figure 3: Color matrices and their relations (3.1) and (3.2) in graphical forms.

where $c=N_{c} / 2$ for a color $S U\left(N_{c}\right)$ group, and $t_{a}$ is the color matrix of the quark in any representation. In particular, if it is in the adjoint representation, then $\left(t_{a}\right)_{b c}=i f_{b a c}$, and this is represented graphically by a triple-gluon vertex read in clockwise order. These relations are shown in Fig. 3 where a cut represents a commutator. Using these figures, decomposition can be accomplished in a graphical way. For details and concrete illustrations, see Ref. [5].

Decomposition of color factor of a cut diagram into regge color factors can be carried out in a similar way, also graphically [7].

A complementary cut diagram with $m-1$ uncut propagators on the upper quark line contains only regge color factors with at most mrg exchanges. This statement is a simple consequence of the graphical construction procedure for the regge color factors [7]. See App. A.

With this simple property, we can now understand why delicate cancellations do not occur in nonabelian cut diagrams. To see that, it is sufficient to show that the spacetime 
amplitude for an $m \mathrm{rg}$ color factor does not have more $\ln s$ power than $g^{2 m}\left(g^{2} \ln s\right)^{p}$, thus there is no need to cancel out higher powers of $\ln s$ to get to the regge behavior. We shall also see that this is not generally so in Feynman diagrams, hence delicate cancellations are necessary there.

Consider a complementary cut diagram with $m-1$ uncut lines. This contains regge color factors with at most $m$ rg exchanges. The corresponding cut diagram for the spacetime amplitude has $m-1$ cut lines. Now each loop in a spacetime diagram can contribute at most one ln $s$ factor, but this factor will be absent in any loop containing a cut propagator. This is so because the Feynman propagator giving rise to the $\ln s$ factor through integration is now replaced by a $\delta$-function [5, 7]. With $m-1$ cut propagators, $m-1$ potential $\ln s$ factors are lost, so the spacetime amplitude can grow as most like $g^{2 m}\left(g^{2} \ln s\right)^{p}$, as claimed.

If these inequalities are saturated, that a spacetime cut diagram with $m-1$ cuts grows like $g^{2 m}\left(g^{2} \ln s\right)^{p}$, then the cut diagram is said to be saturated. Only saturated diagrams are needed in leading-log computations; unsaturated ones are negligible in comparison.

This also means that in the leading-log approximation, there is no need to include in a complementary cut diagram with $m-1$ uncut lines those regge color factors with less than mrg exchanges.

From these discussions we can also see why delicate cancellations are generally expected for Feynman diagrams if the reggeization hypothesis is valid. A Feynman diagram has no cut in its spacetime diagram, nor its color factor. The former tends to give rise to more $\ln s$ factors than a corresponding cut diagram, and the latter will generally yield regge color factors with larger $m$. For both reasons there are too many $\ln s$ powers compared to the reggeized behavior of $g^{2 m}\left(g^{2} \ln s\right)^{p}$, so delicate cancellations eliminating these powers must take place.

\section{$4 s$-channel-ladder cut diagrams}

\subsection{Description of the diagrams}

$s$-channel-ladder diagrams are obtained by joining together the gluons of two quark trees like Fig. 2. If we number the gluons attached to the lower quark tree in the order $[123 \cdots n]$, then the order of gluons along the upper quark tree can be used to specify the whole $s$ channel-ladder diagram. Cut diagrams are determined by the rules discussed in Sec. 2, and cut propagators on the upper quark tree will be indicated by a vertical bar as before. In this notation, Figs. 4(a) to $4(\mathrm{k})$ are respectively $[1|2| 3 \mid 4],[21|3| 4],[31|2| 4],[1|32| 4]$, $[41|2| 3],[1|42| 3],[1|2| 43],[21 \mid 43],[31 \mid 42],[41 \mid 32],[321 \mid 4]$. Unless otherwise stated, propagators along the lower quark tree will remain uncut, so when we refer to cut and uncut propagators, we will usually be speaking of those along the upper quark tree.

We will use the abbreviation SC to denote $s$-channel-ladder cut diagram, and the notation SCC to denote s-channel-ladder complementary cut diagram. The former is used to compute

spacetime amplitudes, and the latter used to compute color factors. Cut propagators in SC become uncut propagators in SCC and vice versa. 


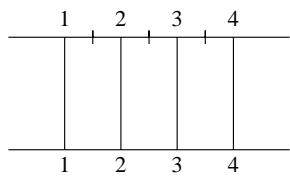

(a)

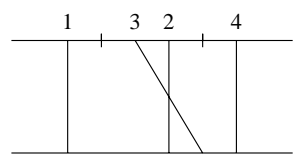

(d)

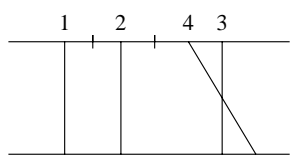

(g)

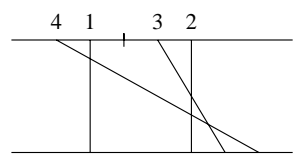

(j)

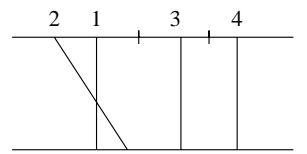

(b)

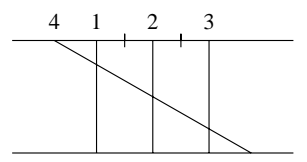

(e)

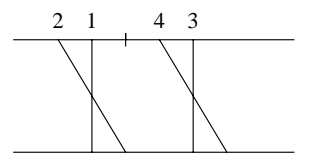

(h)

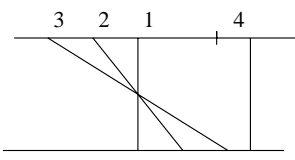

(k)

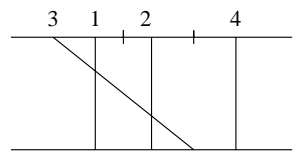

(c)

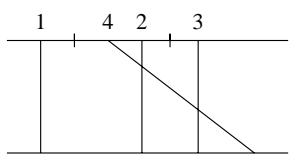

(f)

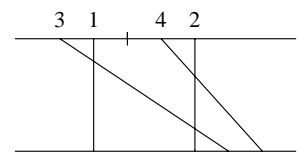

(i)

Figure 4: Examples of 8th order $s$-channel-ladder cut diagrams.

According to the discussion in Sec. 3, in the leading-log approximation, we need to retain only the $m \mathrm{rg}$ regge color factors from a SCC diagram with $m-1$ uncut propagators, and only the saturated SC diagrams with $m-1$ cuts on the upper tree, whose $\ln s$ power is given by $g^{2 m}\left(g^{2} \ln s\right)^{p}$. It is shown in App. B that the saturated SC diagrams are those without adjacent uncut propagators on the upper tree. For example, Figs. 4(a) to 4(j) are saturated, but $4(\mathrm{k})$ is not.

From the rules of Sec. 2, we conclude that all propagators of the planar diagram are to be cut, as in $[1|2| \cdots \mid n]$. For other SC diagrams, cuts are placed behind a number if and only if there is not a smaller number to its right.

We can phrase this in a way independent of the numberings of the gluon lines. All SC diagrams are obtained from the planar diagram by pulling the upper ends of some gluon lines leftward. Once a gluon line is moved, the cut to its right also disappears.

We shall always draw the gluon lines in the planar diagram vertical, as in 4(a). Any other SC diagram will consist of a number of slanted lines mixed in with the vertical lines. The propagator to the right of a slanted line is uncut, but every other propagator is cut. See Fig. 4 for illustrations. The upper ends of two slanted lines in a saturated SC diagram must not be adjacent. In other words, each slanted line will find a vertical line to its right along 
the upper tree, forming together a skeleton cross. These two lines will be called the skeleton lines, and the rest of the vertical lines will be called the mobile lines. Mobile lines have cuts on both sides, or a cut on one side and an external line on the other, while skeleton lines have a cut propagator or an external line on one side, and an uncut propagator on the other. The propagator inside the skeleton cross of a saturated SC is always uncut.

It is the opposite in a saturated SCC. The propagators inside the skeleton crosses are cut but other propagators along the upper quark line remain uncut.

Vertical skeleton lines will be called $v$-lines, slanted skeleton lines will be called $s$-lines, and mobile lines will be called $m$-lines. We shall label the $v, s, m$ lines of a diagram respectively by $v_{i}, s_{i}(1 \leq i \leq k)$, and $m_{j}(1 \leq j \leq b=n-2 k)$. $n$ is the total number of gluon lines and $k$ is the number of skeleton crosses in the diagram.

In terms of these labels, the lower tree of a SC or SCC diagram is a tree in the set

$$
\mathcal{S}_{k, b}^{\prime} \equiv\left\{v_{1} s_{1} ; v_{2} s_{2} ; \cdots ; v_{k} s_{k} ; m_{1} ; m_{2} ; \cdots ; m_{b}\right\}
$$

See Sec. 2 for notations. Conversely, by erecting vertical lines over $v_{i}$ and $m_{j}$, and slanted lines over $s_{i}$ with upper ends lying just to the left of $v_{i}$, we can reconstruct an SC or SCC diagram from any tree in (4.1). However, any permutation of the $k$ skeleton crosses and the $b$ mobile lines will give the same diagram, so the number of distinct diagrams is only $(2 k+b) ! / 2^{k} k ! b$ !. We shall denote the set of distinct diagrams by $\mathcal{S}_{k, b}$. Its trees will be taken from those in $\mathcal{S}_{k, b}^{\prime}$ with $v_{1}<v_{2}<\cdots<v_{k}$, and $m_{1}<m_{2}<\cdots<m_{b}$, where $a<b$ means line $a$ is to the left of line $b$ at the lower end. We shall refer to trees with these restrictions as trees in ascending order.

If we need not know $k$ and $b$ explicitly, we shall simply write the sets as $\mathcal{S}^{\prime}$ and $\mathcal{S}$ respectively.

\subsection{Color factors}

Given a saturated SCC diagram $[T] \in \mathcal{S}$, we want to know how to decompose its color factor into combinations of regge color factors, and conversely given a regge color factor, how to find all the $[T] \in \mathcal{S}$ containing it.

Decomposition is carried out in the following way. The graphical commutation relations in Fig. 3 is used to pull the lower end of the $s$-lines leftward, until it sits just to the right of the corresponding $v$-line, a position which we shall refer to as the home position. As a result, The SCC diagram is given by a sum of many reduced diagrams, each of which having the bottom end of the $s$-lines lying to the left of their original positions, unless of course they were already in their home positions to begin with. Every $s$-line not at its home position should have a cut to its right along the lower tree; an $s$-line at the home position may or may not have such a cut; both are allowed. The sum of all such combinations, weighted with a minus sign iff there is an odd number of cuts, is the decomposition desired. A simple illustration is given in Fig. 5 .

A reduced diagram differs from an SCC diagram in that it is represented by a lower tree with cuts. As discussed above, cuts always occur to the right of $s$-lines, unless they are 

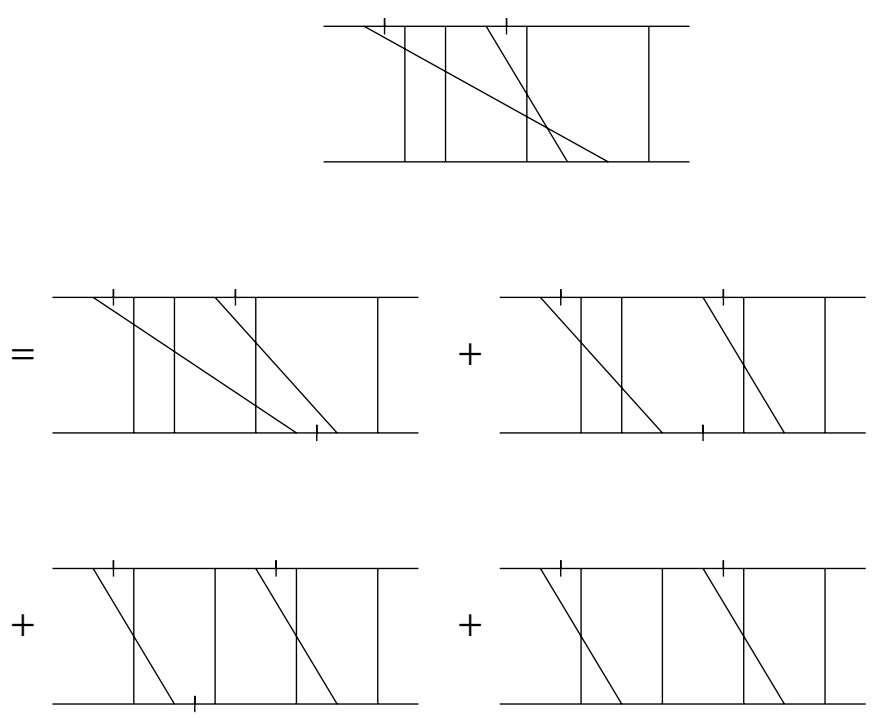

Figure 5: An example of the decomposition of SCC diagrams into sums of reduced diagrams.

already in their home positions. We shall use the symbol $\mathcal{C}$ to denote the set of distinct reduced diagrams, represented by lower cut trees in ascending order.

The color factor of a reduced diagram may or may not be connected after Fig. 3 is applied to get rid of the cuts, and after the upper and lower quark lines are subsequently removed. For example, those in Fig. 6 are connected and the one in Fig. 7 is not. The set of connected diagrams in $\mathcal{C}$ will be denoted by $\mathcal{C}_{0}$.

To judge connectivity one may simply treat the cut in SCC as a device to fuse together the pair of gluon lines it connects. Cuts on the upper tree fuse upper ends of gluon lines, and these are always the skeleton pairs. Cuts on the lower tree fuse lower ends of the gluon lines. If such fusions connect all gluon lines together then the diagram is connected. For example, the reduced diagram $[v s \mid m]$ in $6(\mathrm{c})$ is connected because the skeleton pair $(v s)$ is fused at the upper end and the lines $(\mathrm{sm})$ are fused at the lower end. Similarly, 6(d) is connected. However, the diagram $\left[v_{1} m_{1} s_{1} \mid m_{2} v_{2} s_{2} m_{3}\right]$ in Fig. 7 is not, because fusion leaves it with four components consisting of lines $\left(v_{1} s_{1} m_{2}\right),\left(v_{2} s_{2}\right),\left(m_{1}\right)$, and $\left(m_{3}\right)$.

The color factor of a disconnected reduced diagram is given by the product of the color factor of its connected components, according to the discussion of Sec. 3 and App. A.

The color factor of a connected component may or may not be primitive, depending on whether it is one of those shown in Fig. 1. The color factors in Fig. 6 are primitive, but those in Figs. 8 and 9 are not. However, as is shown in App. A, those that are not all turn out to be zero, so we need not worry about them at least for SCC diagrams.

The only primitive color factors encountered in SCC diagrams are those shown in Fig. 6, and those similar to $6(\mathrm{~d})$ but with $p>2$ skeleton crosses. They have $p$ horizontal lines, with the one to the right always lying at a higher level. These primitive color factors will be denoted as $H_{p}$, with $p=-1,0,1,2, \cdots$ (see Fig. 6), and the corresponding connected 


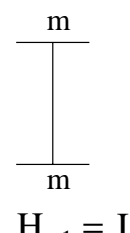

(a)

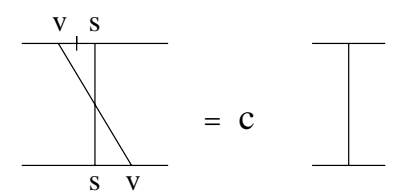

$\mathrm{H}_{0}=\mathrm{c} \mathrm{I}$

(b)

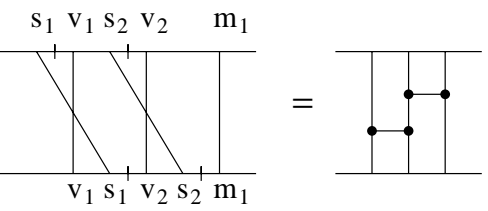

$\mathrm{H}_{2}=\mathrm{H}(+)$

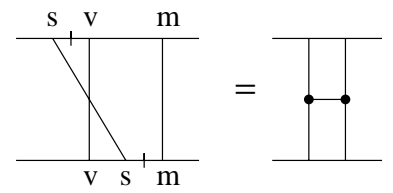

$\mathrm{H}_{1}=\mathrm{H}$

(c)

(d)

Figure 6: Examples of how primitive color factors are obtained from reduced diagrams.

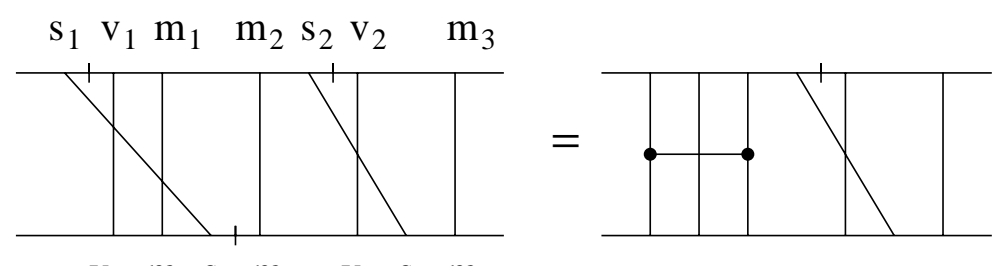

$\begin{array}{llllll}\mathrm{v}_{1} & \mathrm{~m}_{1} \mathrm{~s}_{1} & \mathrm{~m}_{2} & \mathrm{v}_{2} & \mathrm{~s}_{2} & \mathrm{~m}_{3}\end{array}$

Figure 7: An example of a disconnected reduced diagram and the corresponding regge color factor.
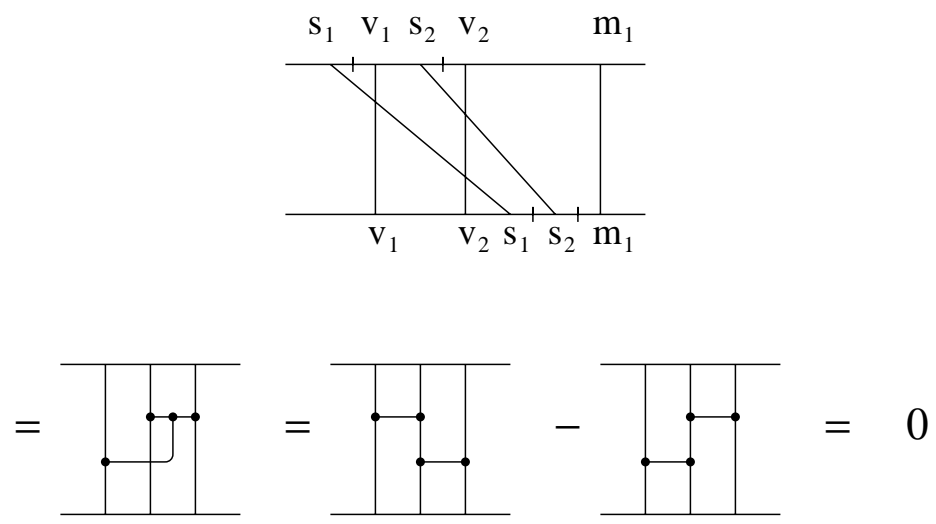

Figure 8: An example of a connected reduced diagram that is not primitive. 

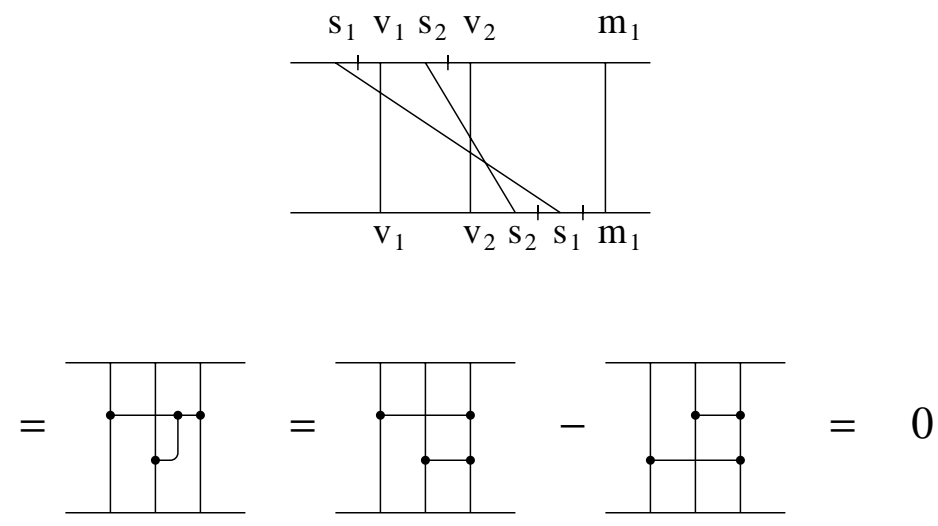

Figure 9: Another example of a connected reduced diagram that is not primitive.

diagrams in $\mathcal{C}_{0}$ by $\left[H_{p}\right]$. It is convenient also to use the designation $I=H_{-1}$ and $H=H_{1}$ because of the similarity of these alphabets to the graphical shape of the diagram. Note that $H_{0}=c H_{-1}=c I$, but all other primitive color factors are independent.

In cut-tree notations, as members of $\mathcal{C}_{0}$, we have

$$
\begin{aligned}
{\left[H_{-1}\right] } & =[m] \\
{\left[H_{0}\right] } & =[v s] \\
{\left[H_{1}\right] } & =[v s \mid m] \\
{\left[H_{2}\right] } & =\left[v_{1} s_{1}\left|m_{1} v_{2} s_{2}\right| m_{2}\right] \\
{\left[H_{p}\right] } & =\left[v_{1} s_{1}\left|m_{1} v_{2} s_{2}\right| m_{2} \cdots v_{p} s_{p} \mid m_{p}\right], \quad(p \geq 1)
\end{aligned}
$$

The reduced diagrams in $\mathcal{C}$ that give rise to a regge color factor $\Phi=\prod_{p \geq-1}\left[(-)^{p} H_{p}\right]^{f_{p}}$ are simply the distinct reduced diagrams obtained by interleaving $f_{p}$ copies of $\left[H_{p}\right]$ together in all possible ways. In symbols,

$$
\{\Phi\}=\left\{\prod_{p}(-)^{p f_{p}} H_{p}^{f_{p}}\right\}=\left\{\left[H_{-1}\right] ; \cdots ;\left[H_{0}\right] ; \cdots ;\left[H_{1}\right] ; \cdots ;\left[H_{2}\right] ; \cdots ; \cdots\right\} 1 / \prod_{p} f_{p} !,
$$

where the ellipses after each $\left[H_{p}\right]$ is an instruction to repeat the same $\left[H_{p}\right] f_{p}$ times, separated by semicolons. The sign $(-)^{p}$ associated with $H_{p}$ comes about because of the minus sign associated with each cut. The notation in (4.3) for interleaving the cut trees in $\mathcal{C}_{0}$ is similar to the notation $\left\{T_{1} ; T_{2} ; \cdots\right\}$ explained in Sec. 2 for interleaving uncut trees $T_{i}$, but with two differences. First, lines separated by cuts are to be thought of as being fused together by the cut, so lines from other cut trees can never be inserted between them. Secondly, each cut diagram in $\left\{\left[H_{p}\right] ; \cdots\right\}$ is going to occur $f_{p}$ ! times because of the identical nature of those diagrams. We allow only distinct diagrams in $\{\Phi\}$ so the division by $\prod_{p} f_{p}$ ! in (4.3) is a formal way of removing such redundancies.

The SCC diagrams in $\mathcal{S}$ that contain the reduced diagrams in $\{\Phi\}$ will be denoted as $\{\Phi\}_{S}$. They can be obtained from the cut trees in $\{\Phi\}$ by getting rid of their cuts, which 
can be accomplished by moving the $s$-lines rightward in all possible ways. Instead of first interleaving the cut trees $\left[H_{p}\right]$ and then getting rid of the cuts, $\{\Phi\}_{S}$ can also be obtained by reversing the two operations, by first getting rid of the cuts and then interleaving the uncut trees, in the following way.

Start from $\left[H_{p}\right] \in \mathcal{C}_{0}$, get rid of the cuts by moving the $s$-lines rightward, to construct all $h_{p}^{i} \in \mathcal{S}(i=1,2, \cdots)$ that reduce to $\left[H_{p}\right]$. In cases like 6(a) to 6(c) where there is only one tree for each $\left[H_{p}\right]$, the degeneracy index $i=1$ will be omitted. This index is however needed in other cases. For example, $\left[H_{2}\right]=\left[v_{1} s_{1}\left|m_{1} v_{2} s_{2}\right| m_{2}\right]$ in $6(\mathrm{~d})$ gives rise to the uncut trees $h_{2}^{1}=\left[v_{1} m_{1} s_{1} v_{2} m_{2} s_{2}\right], h_{2}^{2}=\left[v_{1} m_{1} v_{2} s_{1} m_{2} s_{2}\right], h_{2}^{3}=\left[v_{1} m_{1} s v_{2} m_{2} s_{1} s_{2}\right]$, and $h_{2}^{4}=\left[v_{1} m_{1} v_{2} m_{2} s_{2} s_{1}\right]$. The set of all $h_{p}^{i} \in \mathcal{S}$ for a fixed $p$ will be denoted by $\left\{H_{p}\right\}_{S}$.

The set of distinct SC or SCC diagrams obtained by interleaving $f_{p}$ trees in $\left\{H_{p}\right\}_{S}$ together is just $\{\Phi\}_{S}$.

\subsection{Factorization of sums of spacetime amplitudes}

We proceed to compute the sum of those spacetime amplitudes of all saturated SC diagrams with a common regge color factor $\Phi=\prod_{p}\left((-)^{p} H_{p}\right)^{f_{p}}$. The relevant spacetime diagrams to be summed are those in the set $\{\Phi\}_{S}$.

Using the factorization formula (2.5) on the lower tree, one gets

$$
a\{\Phi\}_{S}=\sum_{[T] \in\{\Phi\}_{S}} a[T]=\prod_{p} \frac{1}{f_{p} !}\left(a\left\{H_{p}\right\}_{S}\right)^{f_{p}}
$$

where

$$
\frac{1}{f_{p} !}\left(a\left\{H_{p}\right\}_{S}\right)^{f_{p}} \equiv \sum \frac{1}{m_{i} !}\left(a\left[h_{p}^{i}\right]\right)^{m_{i}}
$$

with the sum taken over all $m_{i} \geq 0$ subject to $\sum_{i} m_{i}=f_{p}$. Thus $a\left\{H_{p}\right\}_{S}=\sum_{i} a\left[h_{p}^{i}\right]$. The factorials in the denominators of (4.5) arise because of the necessity to keep only distinct diagrams in $\{\Phi\}_{S}$.

The factorization (4.4) and (4.5) for the lower tree can be extended to a factorization for the SC amplitudes. To do so we need to use explicitly the cut property of the upper tree, that the only uncut propagators are those inside the skeleton crosses. To illustrate this point let us look at Fig. 10. Both the (lower) tree [231564] in 10(a) and the tree [253614] in (10b) belong to the set $\{231 ; 564\}$, but if we keep the upper ends of the gluon lines fixed in 10 (a) and 10 (b), permuting the lower ends of the lines to get from 10(a) to 10(b) does not change the SC diagram 10(a) back to another SC diagram. 10(b), with lines 5 and 6 slanting the wrong way, cannot be an SC diagram. However, by making explicit use of the commuting properties of the amplitude for the upper tree, $a[12|3| 45 \mid 6]=a[12] a[3] a[45] a[6]=a[12|45| 3 \mid 6], 10$ (b) can be redrawn as $10(\mathrm{c})$, which is an SC diagram. This can always be done so factorization of the lower tree does lead to a factorization of the sum of saturated SC amplitudes. 


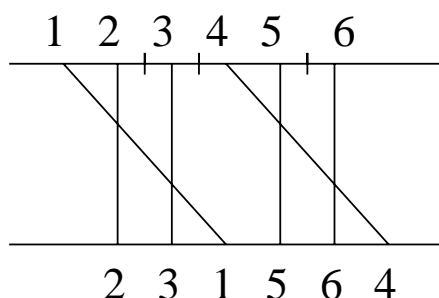

(a)

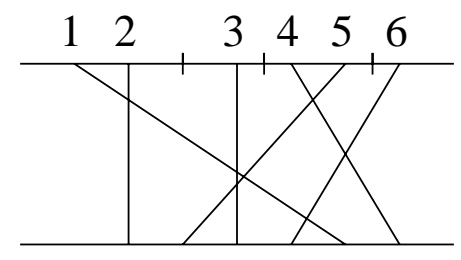

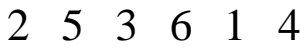

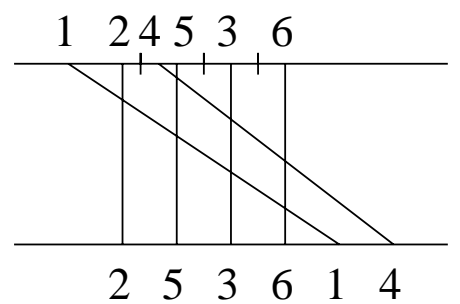

(c)

Figure 10: Two SC diagrams in the set $\{231 ; 564\}$. Diagram $10(\mathrm{~b})$ is identical to diagram $10(\mathrm{c})$.

An SC diagram contains the lower tree, but it also contains gluon propagators, quark propagators along the upper tree, vertex factors, and loop integrations. In lightcone coordinates, $q_{ \pm}=q^{0} \pm q^{3}$, the measure of loop-integration is

$$
\frac{d^{4} q}{(2 \pi)^{4}}=\frac{d^{2} q_{\perp}}{(2 \pi)^{2}} \frac{d q_{+} d q_{-}}{8 \pi^{2}} .
$$

If the Dirac spinors are normalized to $\bar{u} u=1$, and a common factor is taken out of the T-matrix amplitude $\mathcal{T}=-\left(s / 2 M^{2}\right) \mathcal{A}$, then each factorized amplitude $a\left\{H_{p}\right\}_{S}=\sum_{i} a\left[h_{p}^{i}\right]$ corresponds to a saturated SC amplitude $\mathcal{A}\left\{H_{p}\right\}_{S}(\Delta)=\sum_{i} \mathcal{A}\left[h_{p}^{i}\right](\Delta)$, where we have indicated explicitly the dependence on the momentum transfer $\Delta$. The product of two lower-tree amplitude $a\left\{H_{a}\right\} a\left\{H_{b}\right\}$ is turned into convolutions of two SC amplitudes:

$$
\left[\mathcal{A}\left\{H_{a}\right\}_{S} * \mathcal{A}\left\{H_{b}\right\}_{S}\right](\Delta) \equiv(-i) \int \frac{d^{2} q_{\perp}}{(2 \pi)^{2}}\left[\mathcal{A}\left\{H_{a}\right\}\right]\left(\Delta-q_{\perp}\right)\left[\mathcal{A}\left\{H_{b}\right\}\right]\left(q_{\perp}\right) .
$$

In obtaining (4.7), the identity

$$
i \int \frac{d q_{+} d q_{-}}{8 \pi^{2}}(-2 \pi i)^{2} \delta\left(\sqrt{s} q_{+}\right) \delta\left(\sqrt{s} q_{-}\right)(2 s)=-i
$$

has been used.

The sum of all saturated SC amplitudes with the regge color factor $\Phi$ is then given by

$$
\left[\mathcal{A}\{\Phi\}_{S}\right](\Delta)=\prod_{p} \frac{1}{f_{p} !}\left[\mathcal{A}\left\{H_{p}\right\}_{S}\right]^{* f_{p}}(\Delta) .
$$

All the products in (4.9) are meant to be convolutions. In particular, $\left[\mathcal{A}\left\{H_{p}\right\}_{S}\right]^{* f_{p}}$ is taken to mean $f_{p}$ convolutions of the same amplitude. In impact-parameter space, such convolutions is replaced by simple products. 


\section{$4.4 O\left(g^{6}\right)$ results}

Let us now compare the general result of eq. (4.9) with the $O\left(g^{6}\right)$ result of Ref. [7]. Except for the second-order tree diagram, they are shown in Fig. 7 of Ref. [7] as $B 1_{c}, B 2_{c}$, and $C 15_{c}$ to $C 20_{c}$. The spacetime amplitudes are given in eq. (6.1) of that reference were expressed in terms of $\mathcal{M}=-\mathcal{A} / g^{2}$, hence

$$
\begin{aligned}
\mathcal{A}\left(\mathbf{G}_{\mathbf{1}}\right) & =K_{1}-\ln s \frac{c}{2 \pi} K_{2} \\
\mathcal{A}\left(\mathbf{G}_{\mathbf{2}}\right) & =-\frac{1}{2} i K_{2}+i \ln s \frac{c}{2 \pi} K_{3} \\
\mathcal{A}\left(\mathbf{G}_{\mathbf{3}}\right) & =-i \ln s \frac{1}{2 \pi} K_{3} \\
\mathcal{A}\left(\mathbf{G}_{\mathbf{4}}\right) & =-\frac{1}{6} K_{3},
\end{aligned}
$$

where the color factors $\mathbf{G}_{\mathbf{1}}, \mathbf{G}_{\mathbf{2}}, \mathbf{G}_{\mathbf{4}}, \mathbf{G}_{\mathbf{3}}$ are the color factors $I, I^{2}, I^{3}, H$ in the present paper, and where

$$
K_{n}(\Delta)=i^{n-1}\left(* K_{1}\right)^{n}, \quad K_{1}(\Delta)=\frac{g^{2}}{\Delta^{2}} .
$$

The transverse functions $K_{n}$ is related to the ones used in Ref. [7] by $K_{n}=g^{2 n} I_{n}$.

The general result according to (4.9) is

$$
\left[\mathcal{A}\left\{(-H)^{a} I^{b}\right\}_{S}\right](\Delta)=\frac{1}{a ! b !}\left[\mathcal{A}\left\{H_{1}\right\}_{S}\right]^{* a} *\left[\mathcal{A}\left\{H_{-1}\right\}_{S}+c \mathcal{A}\left\{H_{0}\right\}_{S}\right]^{* b}(\Delta) .
$$

Substituting into (4.12) the explicit result obtained from eq. (6.1) of Ref. [7],

$$
\begin{aligned}
\mathcal{A}\left\{H_{-1}\right\}_{S} & =\frac{g^{2}}{\Delta^{2}}=K_{1} \\
\mathcal{A}\left\{H_{0}\right\}_{S} & =-\frac{g^{2}}{c}\left(B 2_{c}\right)=-\ln s \frac{1}{2 \pi} K_{2} \\
\mathcal{A}\left\{H_{1}\right\}_{S} & =-g^{2}\left(C 20_{c}\right)=\ln s \frac{i}{2 \pi} K_{3},
\end{aligned}
$$

we get

$$
\left[\mathcal{A}\left\{(-H)^{a} I^{b}\right\}_{S}\right](\Delta)=\frac{1}{a ! b !}\left[\ln s \frac{i}{2 \pi} K_{3}\right]^{* a} *\left[K_{1}-\ln s \frac{c}{2 \pi} K_{2}\right]^{* b}(\Delta) .
$$

The color factors $\mathbf{G}_{\mathbf{1}}, \mathbf{G}_{\mathbf{2}}, \mathbf{G}_{\mathbf{4}}$ correspond to $(a, b)=(0,1),(0,2),(0,3)$, and the color factor $\mathbf{G}_{\mathbf{3}}$ corresponds to $(a, b)=(1,0)$ but with an extra minus sign. Expanding (4.14), keeping only leading- $\log$ contributions and only up to $O\left(g^{6}\right)$, the result is the same as $(4.10)$. 


\subsection{Reggeized factorization}

The expression $\mathcal{A}\left\{H_{-1}\right\}_{S}+c \mathcal{A}\left\{H_{0}\right\}_{S}=K_{1}-(c / 2 \pi) \ln s K_{2}$ in (4.12) and (4.14) is the first two terms of the reggeized-gluon propagator

$$
R_{1}(\Delta, s)=\frac{g^{2}}{\Delta^{2}} \exp (-\bar{\alpha}(\Delta) \ln s), \quad \bar{\alpha}(\Delta)=\frac{c}{2 \pi g^{2}} \Delta^{2} K_{2}(\Delta) .
$$

The other terms come from $t$-channel-ladder and associated diagrams [2, 3, 5] not considered here. The term $\mathcal{A}\left\{H_{1}\right\}_{S}$ in (4.12) is one of the many terms contributing to the emission and reabsorption of an ordinary gluon from a reggeized gluon, as indicated by the pattern $\mathrm{H}$. Even to $O\left(g^{6}\right)$, it receives contributions from other diagrams as well [0]. When all these are taken into account, it is known that such emission and absorption can be constructed from the Lipatov vertex [2] .

So the factorized results (4.9) and (4.12) are the beginning of contributions that lead to reggeized factorization, but the reggeization property cannot be seen fully without including other diagrams. However, the reggeized nature of the color factors does seem to emerge rather naturally.

\section{$5 \quad$ Summary and outlook}

In this paper we initiated a leading-log investigation on sums of Feynman diagrams contributing to multiple reggeized-gluon exchanges. These diagrams are important because they supply nonleading-log contributions to the $S U(3)_{c}$ gluon and Pomeron amplitudes, thereby restoring unitarity. In any case they supply the leading contributions in an $S U\left(N_{c}\right)$ color theory in which the colliding beams carry large color so they must be taken into account.

The central question studied in this paper is whether sums of Feynman diagrams in the leading-log approximation will factorize into multiple-reggeon-exchange diagrams as depicted in Fig. 1. This 'reggeized factorization hypothesis' is nontrivial to prove for at least two reasons. First, it is known that there are delicate cancellations in sums of Feynman diagrams, so individual diagrams must be computed to subleading-log accuracy to ensure a finite contribution to the sum. This is a very difficult task for high order diagrams. Secondly, high-order Feynman diagrams are very complicated, with lines criss-crossing in a complex pattern, so it is far from obvious that they will sum up and factorize into neat patterns as those displayed in Fig. 1. To date, factorization had been proved completely only to the 6 th order, and partially to the 8th and 10th orders, by explicit calculations.

We prove in this paper the reggeized factorization hypothesis for $s$-channel-ladder diagrams of any complexity. Both of the difficulties mentioned above are solved by using instead the technique of nonabelian cut diagrams discussed in a previous publication. These cut diagrams are resummations of Feynman diagrams and are different from the Cutkosky cut diagrams.

For other diagrams the validity of the reggeized factorization hypothesis is still under investigation. 


\section{Acknowlegements}

We thank Jean-René Cudell and Omid Hamidi-Ravari for interesting discussions. This research is supported in part by the by the Natural Science and Engineering Research Council of Canada, and the Fonds pour la Formation de Chercheurs et l'Aide à la Recherche of Québec, and YJF wishes to acknowledge the support of the Carl Reinhart Foundation.

\section{A Color factors of nonabelian cut diagrams}

Color factor of nonabelian cut diagrams are calculated using the graphical rules in Fig. 3. Some explicit examples are shown in Figs. 6-9. In what follows, we shall discuss some of the general properties in the leading-log approximation.

Fig. 3(c) can be used to get rid of cuts on the complementary cut diagrams. As a result, diagrams with $m-1$ uncut propagators along the upper tree has at most $m$ gluon lines attached to it. We say 'at most', because relations 3(b), 3(d), and 3(e) can sometimes be used to get rid of more lines.

Since cuts are made on the upper tree, the number of gluons $n$ attached to the lower tree is often larger than the number $m$ attached to the upper tree. However, by using Fig. 3 again to manipulate the lines attached to the lower tree, at least in all cases encountered in Sec. 4, one can reduce the lines attached to the lower tree to be $m$. Hence complementary cut diagrams with $m-1$ uncut propagators along the upper tree contribute color factors with $m$-reggeized-gluons, or less. It was then argued in Sec. 3 of the text that we need not keep those with less than $m$ reggeons in the leading-log approximation.

It is conceivable, for very complicated diagrams, that we cannot reduce $n$ to $m$ with the rules of Fig. 3 alone. The resulting color factor has $n \neq m$, so it cannot possibly contribute if Fig. 1 is the final result. For that reason we shall define the leading-log approximation to exclude all such color factors that cannot be reduced to $n=m$.

Using 3(b) and 3(c) again, the positions of gluon lines attached to the upper or the lower tree can be reversed; their difference being a diagram with one less gluon line attached to the upper/lower tree, and hence can be ignored in the leading-log approximation. This is why primitive color factors can cross one another in any way along the upper and the lower trees, yet giving exactly the same result in the leading-log approximation.

Finally, we want to prove that any color factor with an $s$-line climbing onto the underside of a horizontal line, like those found in Figs. 8 and 9, would be zero. The proof is shown in Fig. 11, where the shaded area can contain a very complicated structure. Use 3(b) and 3 (c) (for four gluon lines) to move the point joining the bottom of the horizontal line to the right, one gets 11(a) and 11(b). Moving that point to the left, one gets 11(c) and 11(d). Within the leading-log approximation, we can pull the middle vertical line of 11(c) to the extreme right to get $11(b)$, hence $11(b)=11(c)$. Similarly $11(a)=11(d)$. Therefore $11(\mathrm{a})-11(\mathrm{~b})=-[11(\mathrm{a})-11(\mathrm{~b})]=0$. 


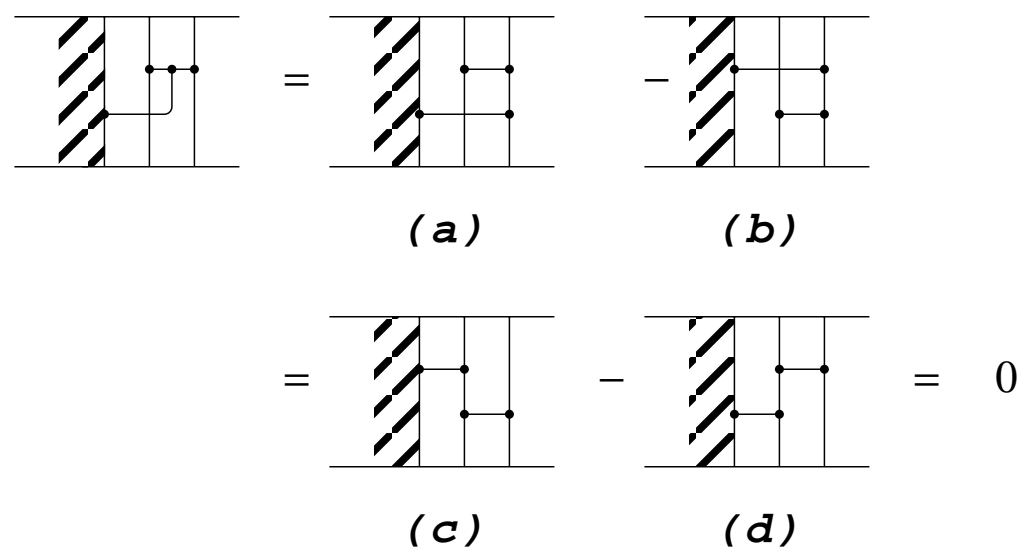

Figure 11: Proof that an SCC diagram cannot yield a non-vanishing connected diagram that is not primitive. Within the leading-log approximation, $11(\mathrm{c})=11(\mathrm{~b})$ and $11(\mathrm{~d})=11(\mathrm{a})$.

\section{B Saturated ladder diagrams}

We want to show in this Appendix that an SC diagram (see Sec. 4 for notation) with two adjacent uncut propagators is unsaturated. By definition, a saturated diagram of $(2 n)$ th order and $m \mathrm{rg}$ exchange $\left(m-1\right.$ cut lines) have a $g$ and $s$ dependence $g^{2 m}\left(g^{2} \ln s\right)^{n-m}$. An unsaturated diagram is one with a slower $s$ growth in comparison.

Ref. [7] contains explicit calculations to $O\left(g^{6}\right)$. By examining Fig. 7 and eq. (6.1) of that reference, it can be seen that this assertion is valid to $O\left(g^{6}\right)$. If one now follows the calculation of these examples with the method of Ref. [5] and Appendix B of Ref. [7], one can see that these calculations can easily be generalized to a multiloop situation as follows.

Consider an SC diagram with $n=l-1$ gluon lines. Let $q_{i}=\left(q_{i+}, q_{i-}, q_{\perp}\right)(1 \leq i \leq l)$ be the gluon momenta in the lightcone coordinates, and $q_{i-} \equiv x_{i} \sqrt{s}$.

We shall follow Ref. [5] by calculating the high energy behavior using residue calculus and flow diagrams to carry out the ' + ' integrations.. For SC diagrams without adjacent uncut propagators, there is a unique flow path for each diagram, and the poles for the '+' integration can always be taken along the lower tree. The gluon propagators are then $\sim 1 / q_{i \perp}^{2}$, and considered to be $O(1)$. This leaves the uncut propagators along the upper tree to the '-' integration, each of which contributes to a factor of $\ln s$ via '-' integration of the type $\int_{s^{-1}} d x_{i} / x_{i}$. Hence such diagrams have their full share of $\ln s$ factors and are saturated.

For diagrams with two adjacent uncut propagators, the flow path is never unique: the flow direction along the boundary of the two adjacent uncut loops cannot be determined. See Figs. 10.7 and 10.8 of Ref. [5] for concrete examples. As a result, at least one pole from the ' + ' integration must not come from the lower tree. Explicit calculation then shows that such diagrams are at least one $\ln s$ power down from the saturated ones.

The origin of this reduction can be seen as follows. The ' + ' momentum is inversely proportional to the ' - ' momentum at the poles. Elsewhere the ' + ' momenta are determined by momentum conservation. Now the '-' momentum flows predominantly along the lower 
tree, so if the pole is off it on a gluon line, the ' + ' momentum flowing through that line would be relatively large. By momentum conservation, there must be a return flow passing through part of the lower tree and another gluon line, and the Feynman propagators of these are large because of the large ' + ' flow through them. This brings about at least two small factors $x_{i}$, overcompensating the large factor $1 / x_{i}$ from the residue of the pole. This costs at least a $\ln s$ factor to be lost from the '-' integration. Hence the diagram is unsaturated.

\section{References}

[†] Electronic address: feng@physics.mcgill.ca

[*] Electronic address: lam@physics.mcgill.ca

[1] F.E. Low, Phys. Rev. D12 (1975) 163; S. Nussinov, Phys. Rev. Lett. 34 (1975) 1286.

[2] L.N. Lipatov, Yad. Fiz. 23 (1976) 642 [Sov. J. Nucl. Phys. 23 (1976) 338]; Ya. Ya. Balitskii and L.N. Lipatov, Yad. Fiz. 28 (1978) 1597 [Sov. J. Nucl. Phys. 28 (1978) 822]; E.A. Kuraev, L.N. Lipatov, and V.S. Fadin, Zh. Eksp. Teor. Fiz. 71 (1976) 840 [Sov. Phys. JETP 44 (1976) 443]; ibid. 72 (1977) 377 [ibid. 45 (1977) 199]; V. Del Duca, hep-ph/9503226.

[3] C.Y. Lo and H. Cheng, Phys. Rev. D13 (1976) 1131; D15 (1077) 2959; H. Cheng, J.A. Dickinson, and K. Olaussen, Phys. Rev. D23 (1981) 534.

[4] See, e.g., V.S. Fadin, R. Fiore, and M.I. Kotsky, hep-ph/9605357.

[5] H. Cheng and T.T. Wu, 'Expanding Protons: Scattering at High Energies', (M.I.T. Press, 1987).

[6] N.T. Nieh and Y.P. Yao, Phys. Rev. Lett. 32 (1974) 1074; Phys. Rev. D13 (1976) 1082; B. McCoy and T.T. Wu, Phys. Rev. Lett. 35 (1975) 604; Phys. Rev. D12 (1975) 3257; D13 (1976) 1076; L. Tyburski, ibid. 13 (1976) 1107; L.L. Frankfurt and V.E. Sherman, Yad. Fiz. 23 (1976) 1099 [Sov. J. Nucl. Phys. 23 (1976) 581]; A.L. Mason, Nucl. Phys. B117 (1976) 493.

[7] Y.J. Feng, O. Hamidi-Ravari, and C.S. Lam, to appear in Phys. Rev. D (McGill/96-13, hep-ph/9604429).

[8] C.S. Lam and K.F. Liu, to be published (McGill/96-12, hep-ph/9604337).

[9] H. Cheng and T.T. Wu, Phys. Rev. 186 (1969) 1611; M. Levy and J. Sucher, Phys. Rev. 186 (1969) 1656. 